\title{
Mycobacterium tuberculosis infection within a parotid Warthin tumor: Magnetic resonance imaging appearance
}

\author{
Chia Han Lin, Mu Kuan Chen
}

\begin{abstract}
Introduction: Parotid tuberculosis is a rare disease, accounting for less than $1 \%$ cases of extra-pulmonary tuberculosis. Misdiagnosis is common when a coexisting parotid tumor is present, a diagnosis of coexisting parotid tuberculosis and a Warthin tumor usually requires multiple imaging examinations, however some studies have reported the use of magnetic resonance imaging (MRI) prior to surgical intervention. Case Report: We report the case of a 72-year-old male with Mycobacterium tuberculosis infection within a Warthin tumor. We performed complete superficial parotidectomy and postoperative anti-tuberculosis treatment was given. The patient was regularly followed-up for more than six months, and no recurrence was noted. Conclusion: We reviewed the advantages and disadvantages of MRI. An MRI is quite sensitive in diagnosing granulomatous disease, but quantitative measurement for specific disease is needed through large series of study in the future.
\end{abstract}

Chia Han Lin ${ }^{1}$, Mu Kuan Chen ${ }^{2}$

Affiliations: ${ }^{1} \mathrm{MD}$, Fellowship, Otorhinolaryngology \& Head and Neck Surgery, Changhua Christian Hospital, Changhua City, Taiwan, Republic of China; ${ }^{2} \mathrm{MD}, \mathrm{PhD}$, Chief and Medical Director, Ororhinolaryngology \& Head and Neck Surgery and Oral Cancer Research Center, Changhua Christian Hospital, Changhua City, Taiwan, Republic of China.

Corresponding Author: Chia Han Lin Room 110, No.135, St. Nanxiao, Changhua City, Taiwan, Republic of China. 500; Ph: +886-4-7238595-7210; Fax No: 886-4-7232492; Email: 135196@cch.org.tw

Received: 06 December 2012

Accepted: 13 May 2013

Published: 01 July 2013
Keywords: Warthin tumor, Parotid abscess, Mycobacterium tuberculosis, Anti-tuberculosis

$* * * * * * * * *$

Lin $\mathrm{CH}$, Chen MK. Mycobacterium tuberculosis infection within a parotid Warthin tumor: Magnetic resonance imaging appearance. International Journal of Case Reports and Images 2013;4(7):363-367.

$$
* * * * * * * * *
$$

doi:10.5348/ijcri-2013-07-332-6

\section{INTRODUCTION}

Parotid tuberculosis is a rare type of extrapulmonary tuberculosis. It usually presents as a unilateral swelling or abscess involving the parenchyma of the gland either through hematogenous spread or from infection of the lymph nodes within or around it. A Warthin tumor is a benign parotid tumor which is clinically diagnosed by computed tomography (CT), magnetic resonance imaging (MRI), fine needle aspiration, and excision biopsy. Parotid tuberculosis rarely coexists with Warthin tumor and is indistinguishable on most CT scan. Thus, we chose MRI to attempt to distinguish the differences between parotid tuberculosis and Warthin tumor.

Complete superficial parotidectomy and postoperative anti-tuberculosis medical treatment are usually sufficient in such cases. After complete treatment, the recurrence rate of these rare coexisting diseases is quite low.

\section{CASE REPORT}

A 72-year-old man was presented to our clinic with a left cheek swelling mass with mild tenderness for one month. He was a heavy smoker with at least two packs 
of tobacco per day. Clinically, the tumor was located inferior to the left auricle; with elastic, tactile, movable content, and was well capsulated. No other palpable neck mass or facial palsy was noted, and there were no overlying skin changes suggestive of parotiditis. An oral cavity examination revealed no abnormal findings. A flexible nasopharyngoscopic examination revealed negative pathological findings of the nasopharynx, oropharynx, hypopharynx, and larynx. Head and neck T2-weighted MRI scan showed a 3×3.7 cm high intensity mass within the left superficial parotid gland. Small cervical lymphadenopathies were also apparent in bilateral level II and I. In T2-weighted image, left parotid high signal with central necrosis suggestive of left pleomorphic adenoma or mixed tumor (Figure 1). In T1-weighted image post contrast infusion, left well demarcated parotid mass was moderated enhanced, with some low to moderate signal and heterogenous material inside. Highly suggestive of tumor central bleeding or granulomatous disease (Figure 2). In T2weighted image, left parotid tumor shows high signal with central necrosis, suggestive of granulomatous disease (Figure 3).

Left superficial parotidectomy was then performed under general anesthesia. During dissection, multiple granulation tissues with fibrosis surrounding the mass resulted in difficulty in dissecting the tumor and identifying the facial nerve. On gross examination, a

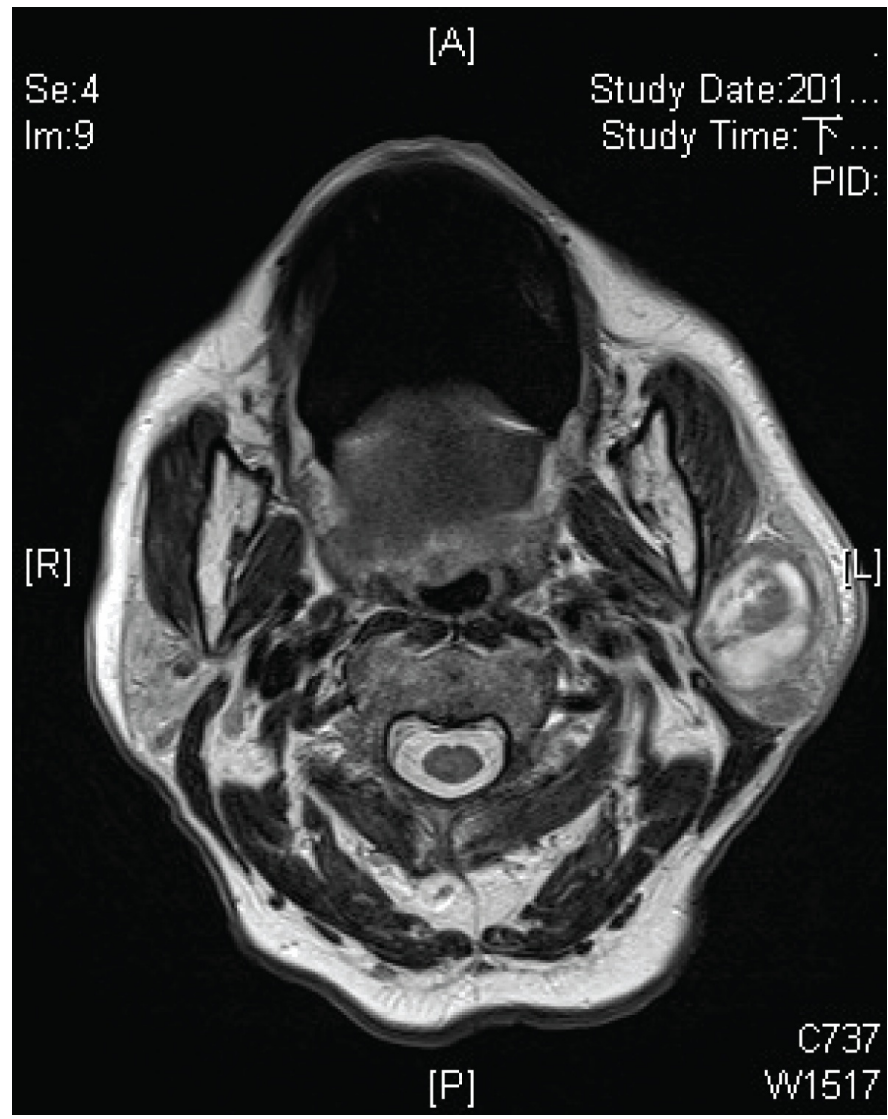

Figure 1: In T2-weighted image, left parotid high signal with central necrosis suggestive of left pleomorphic adenoma or mixed tumor.

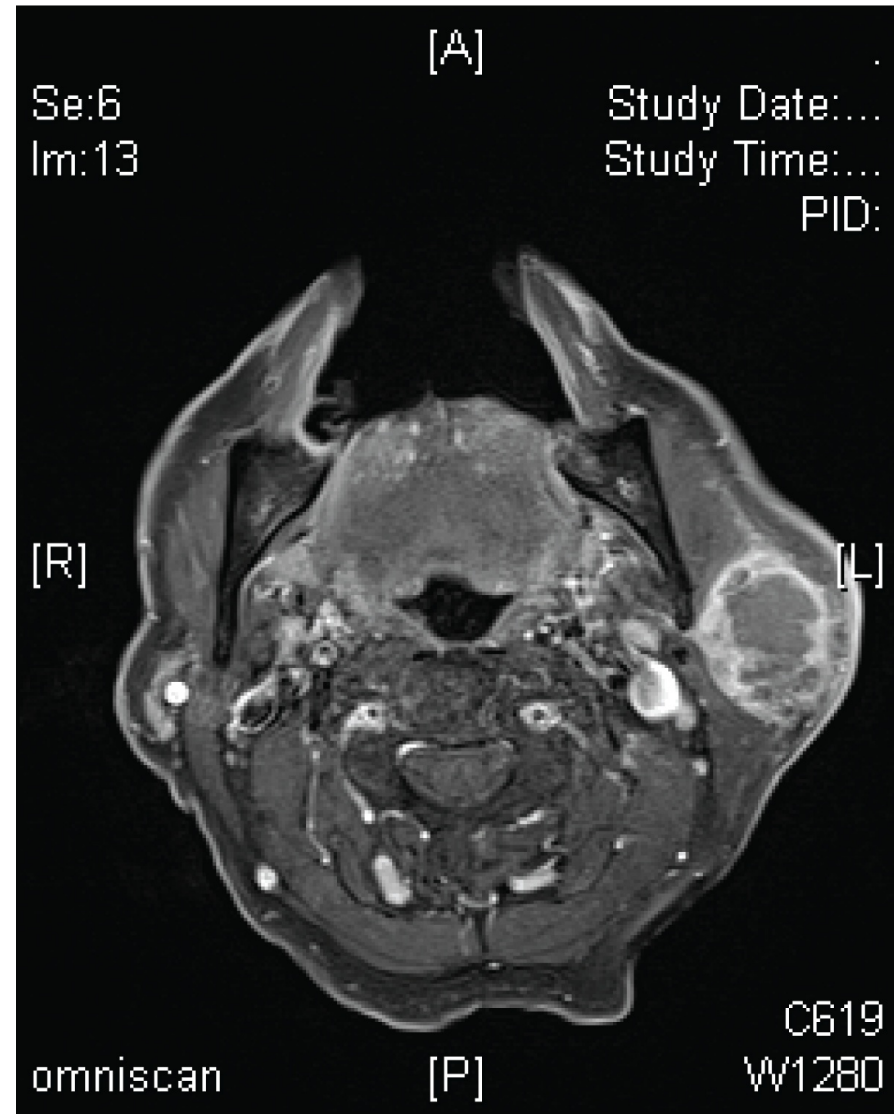

Figure 2: In T1-weighted image post contrast infusion, left well demarcated parotid mass was moderated enhanced, with some low to moderate signal and heterogenous material inside. Highly suggestive of tumor central bleeding or granulomatous disease.

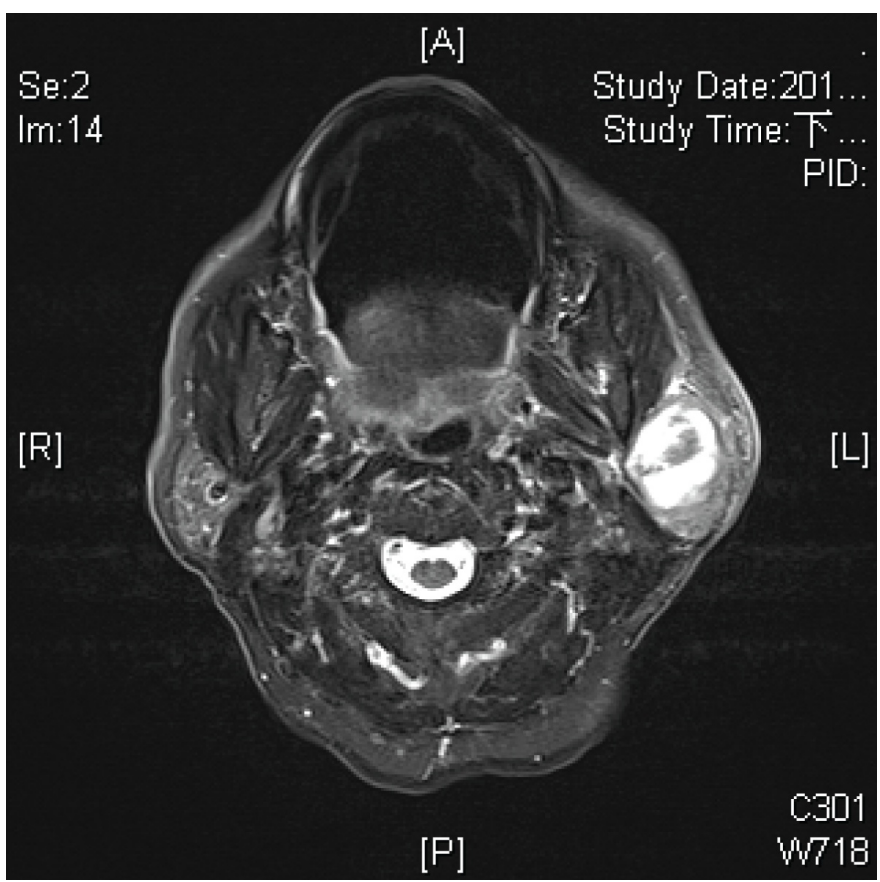

Figure 3: In T2-weighted image, left parotid tumor showing high signal with central necrosis, suggestive of granulomatous disease. 
$3.1 \mathrm{x} 4 \mathrm{~cm}$ tumor was found with some caseous necrotic material bursting out. Pathology revealed a Warthin tumor with chronic inflammation and multiple granulation. No malignant tissue was found. The histopathological diagnosis was a small Warthin tumor with Mycobacterium bacilli within the tumor (Figures 4

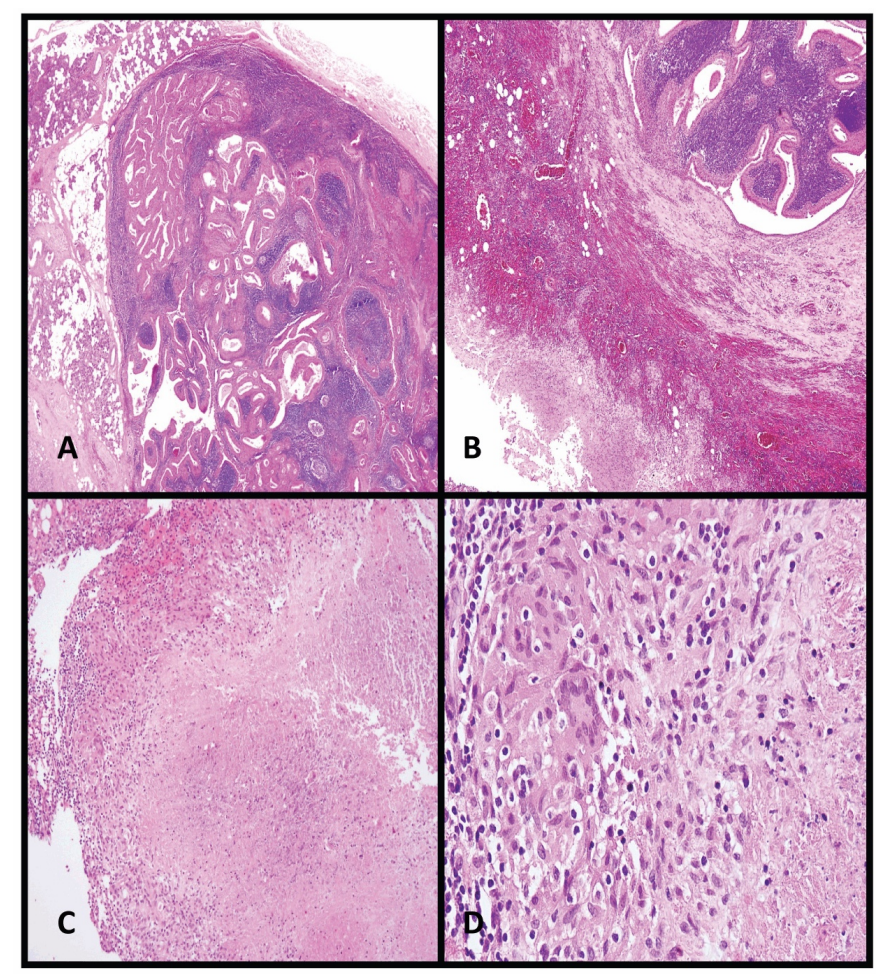

Figure 4: (A) A small Warthin tumor composed of papillary architectures lined by two layers of oncocytic epithelium with surrounding lymphoid stroma is seen, (B) Foci of caseating granulomatous inflammation and granulation tissue adjacent to the Warthin tumor are present, (C) A well-formed granuloma with epithelioid macrophages surrounding caseating necrosis is seen, (D) High power view illustrating aggregates of epithelioid macrophages with a multinucleated giant cell surrounding the caseating necrosis.

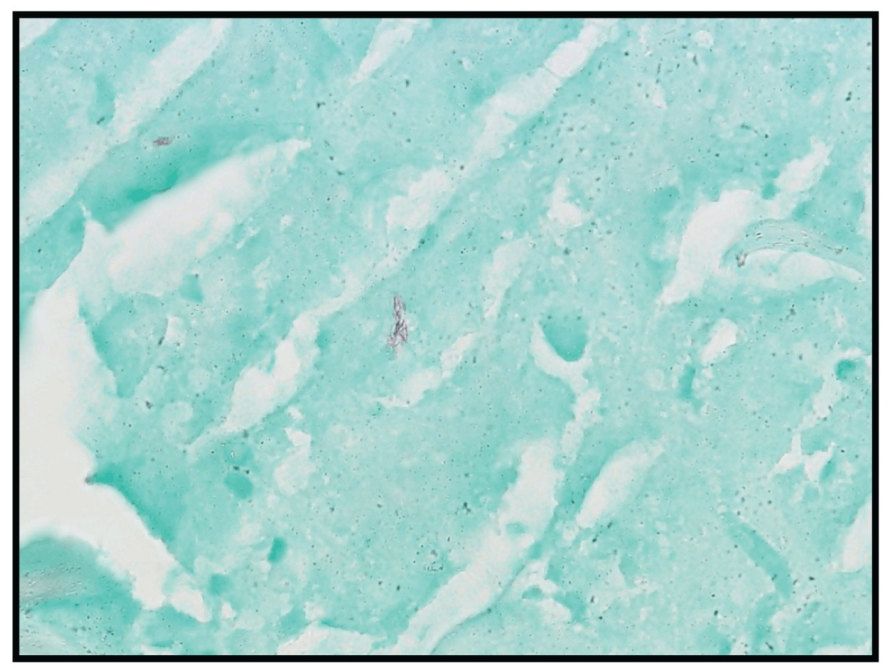

Figure 5: Mycobacteria within the caseating necrosis are seen in Kinyoun acid fast stain (x1000). and 5). The Mycobacterium culture revealed Mycobacterium tuberculosis. His wound recovered well and there was no facial palsy. Anti-tuberculosis therapy was then given for three months. The dosage and the duration was as follows: rifampin $600 \mathrm{mg}$, isoniazid 600 $\mathrm{mg}$, pyrazinamide $1500 \mathrm{mg}$, ethambutol $1200 \mathrm{mg}$ once daily for three months. The patient has been regularly followed-up for renal function, liver function, and hearing function, and there was no abnormality in the above examination nor local recurrence to date for three years.

\section{DISCUSSION}

Parotid gland lesions may occur due to various disease such as sialolithiasis, sialoadenitis, and benign or malignant tumors. The diagnostic approach includes CT scan, MRI scan, fine needle aspiration, and excision biopsy, and histopathology is used to make a definite diagnosis.

Extra pulmonary tuberculosis represents approximately $25 \%$ of overall tubercular morbidity. The most common type of extra pulmonary tuberculosis is lymph node tuberculosis, and other forms include pleural, skeletal, central nervous system, abdominal, genitourinary, miliary, and tubercular pericarditis [1]. Parotid tuberculosis is a rare form of extra pulmonary tuberculosis. It usually presents as a unilateral swelling or abscess involving the parenchyma of the gland either through hematogenous spread or from infection of lymph nodes within or around it. It presents as a slow growing mass and is mostly indistinguishable from parotid tumors, and it may also coexist with a parotid tumor [2]. Tuberculosis comprises $2.5-10 \%$ of parotid lesions. If there are no clinical pulmonary symptoms, it is difficult to make a diagnosis [3].

Other infectious processes such as fungal infections are also seen in parotid abscesses [4]. Fungal infections are quite rare in parotid infections. However, when patient is immunocompromised, fungal infections should always be kept in mind. The patients are generally critically ill, especially when coexisting with a Warthin tumor. Adequate antifungal therapy and incision and drainage is crucial.

De Paoli reported the first case of parotid gland tuberculosis in 1893. The most common route of infection of the parotid gland is by direct extension of bacilli from the oral cavity via the gland ductal system. Watanabe et al. first reported a patient with mycobacterial infection with a Warthin tumor confirmed by polymerase chain reaction [5]. However, preoperative imaging was often unavailable in the past, or poor resolution of the images may have lead to difficulty in making a diagnosis.

Ultrasonography may have been the first imaging modality of choice for the assessment of palpable abnormalities of the parotid gland. Ultrasonography is able to differentiate possible benign from malignant neoplasms. In addition, fine needle aspiration can also be performed with ultrasound for pathologic tissue 
proof [6]. However, ultrasonography is a skill dependent examination method.

Computed tomography scan then became the preferred choice due to the higher resolution. Wei et al. reported the performance of CT scan in detecting tuberculosis infections of the parotid gland [7]. The main feature of parotid tuberculosis on CT is multiple nodularity. Another differential diagnosis may include sarcoidosis-a systemic disease often presenting as noncaseating granuloma bilaterally. CT scan is most sensitive and specific in identifying bony erosions caused by tumors. However, this seldom occurs with Warthin tumors or tuberculosis infection of the parotid gland.

An MRI scan may be better than CT scan in differentiating parotid from extra-parotid space masses, such as paraganglioma, schwannoma, or minor salivary gland masses. Magnetic resonance imaging also provides for better evaluation of tumors invading the parapharyngeal or intracranial space. On T1-weighted images, almost all parotid masses are isointense to muscle, and can be separated from the normal hyperintense signal intensity of the rest of the parotid gland. On fat-saturated T1-weighted post-contrast images of the tumor, these sequences may be used to determine the boundary and extent of the lesion. In general, T2-weighted images are not specific in differentiating tumor types, as most tumors are hyperintense in signal intensity. However, lesions that demonstrate iso- to hypo-intense signal intensity relative to muscle may be indicative of malignancy. MRI is relatively artifact free and does not use ionizing radiation, and multiple planes are readily obtained without changing the position of the patient. The appearance of tuberculosis on MRI is that of a central water-density collection (i.e., low or medium signal intensity on T1-weighted images, with increased signal intensity on T2-weighted images) in a bed of edema [8]. Pleomorphic adenoma on MRI demonstrates a predominantly heterogeneous well-circumscribed mass of intermediate to low signal intensity on T1-weighted images, and hyperintense signal intensity on T2weighted sequences. Hemorrhage is revealed as areas of high-intensity signals on T1- and T2-weighted images. It is difficult to differentiate pleomorphic adenoma from tuberculosis infection. Even though the appearance of tuberculosis on MRI shows central edematous and fluid accumulation, this is also seen in Warthin tumors. Since these two diseases are hyperintense in signal intensity. It is especially difficult to differentiate them when they coexist. However, MRI scan is still the gold standard in differentiating parotid diseases. Careful clinical history taking and thorough physical examinations are crucial to avoid a misdiagnosis, despite the use of advanced technology. An MRI scan is contraindicated for patients with claustrophobia, pacemakers, intracranial ferromagnetic aneurysm clips, cochlear implants and brainstem implants placed for hearing loss, ocular foreign bodies, and surgical ferromagnetic hardware.

Four drug regimens (rifampicin, isoniazid, ethambutol and pyrazinamide) in the intensive phase followed by two drugs (rifampicin and isoniazid) in the continuation phase are recommended for parotid tuberculosis. However, a regimen consisting of only three drugs (rifampicin, isoniazid and pyrazinamide) followed by two drugs (rifampicin and isoniazid) may also be sufficient, as parotid tuberculosis is a paucibacillary extra pulmonary form of tuberculosis.

\section{CONCLUSION}

This report should alert clinicians to the importance and difficulties in diagnosing coexisting Warthin tumors and parotid tuberculosis. Magnetic resonance imaging is still the preferred imaging modality to diagnose parotid lesions. Future research on the quantitative values of the magnetic resonance signals for parotid lesions is warranted to allow for the correct diagnosis by using cut-off values rather than subjective descriptions.

$* * * * * * * * *$

\section{Author Contributions}

Chia Han Lin - Substantial contributions to conception and design, Acquisition of data, Analysis and interpretation of data, Drafting the article, Revising it critically for important intellectual content, Final approval of the version to be published

$\mathrm{Mu}$ Kuan Chen - Analysis and interpretation of data, Drafting the article, Revising it critically for important intellectual content, Final approval of the version to be published

\section{Guarantor}

The corresponding author is the guarantor of submission.

\section{Conflict of Interest}

Authors declare no conflict of interest.

\section{Copyright}

(C) Chia Han Lin et al. 2013; This article is distributed under the terms of Creative Commons Attribution 3.0 License which permits unrestricted use, distribution and reproduction in any means provided the original authors and original publisher are properly credited. (Please see www.ijcasereportsandimages.com /copyright-policy.php for more information.)

\section{REFERENCES}

1. Garg R, Verma SK, Mehra S, Srivastawa AN. Parotid tuberculosis. Lung India 2010 Oct;27(4):253-5.

2. Archontaki M, Stamou AK, Hajiioannou JK, Kalomenopoulou M, Kyrmizakis DE. Intraparotid lymph node tuberculosis. B-ENT 2008;4(1):35-7.

3. Ozcan C, Apa DD, Aslan G, Gülhan S, Görür K. Mycobacterium tuberculosis infection within parotid gland Warthin tumor. J Craniofac Surg 2008 Nov;19(6):1561-5. 
4. Leibowitz JM, Montone KT, Basu D. Warthin tumor presenting as a fungal abscess in an immunocompetent host: case report and review of the literature. Head Neck 2010 Jan;32(1):133-6.

5. Watanabe $M$, Nakayama $T$, Koduka $Y$, et al. Mycobacterium tuberculosis infection within Warthin's tumor: report of two cases. Pathol Int 2001 Oct;51(10):797-801.

6. Bagga P, Pandey P, Shahi M, Mittal A, Mehta V, Ganju A. Parotid gland tuberculosis diagnosed on FNAC: a case report. Cytopathology 2010 Apr;21(2):127-9.

7. Wei Y, Xiao J, Pui MH, Gong Q. Tuberculosis of the parotid gland: computed tomographic findings. Acta Radiol 2008 May;49(4):458-61.

8. Birkent H, Karahatay S, Akcam T, Durmaz A, Ongoru O. Primary parotid tuberculosis mimicking parotid neoplasm: a case report. J Med Case Reports 2008 Feb 26;2:62.

Access full text article on other devices

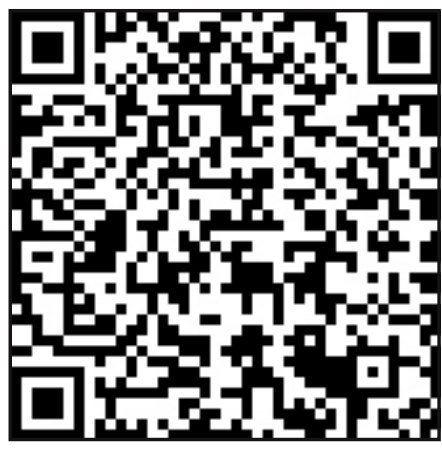

Access PDF of article on other devices

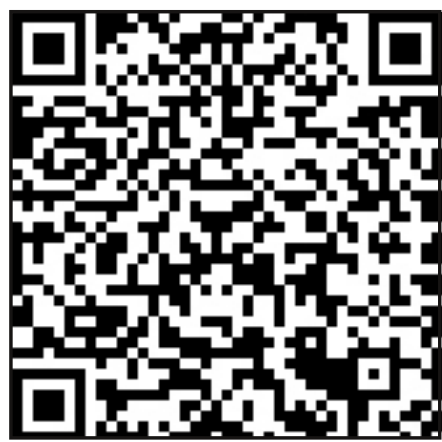

